EOMmun Communication et organisation

Or

Les nouvelles cultures de l'information dans les organisations

\title{
Les cadres et le numérique
}

Cultures informationnelles et sentiment d'efficacité

Managers and digital issues: Informational cultures and feeling of efficiency

Aurélia Dumas, Fabienne Martin-Juchat et Julien Pierre

\section{(2) OpenEdition}

Journals

Édition électronique

URL : http://journals.openedition.org/communicationorganisation/5520

DOI : $10.4000 /$ communicationorganisation. 5520

ISBN : 979-10-300-0146-4

ISSN : $1775-3546$

Éditeur

Presses universitaires de Bordeaux

Édition imprimée

Date de publication : 1 juin 2017

Pagination : 55-66

ISBN : 979-10-300-0162-4

ISSN : $1168-5549$

Référence électronique

Aurélia Dumas, Fabienne Martin-Juchat et Julien Pierre, "Les cadres et le numérique »,

Communication et organisation [En ligne], 51 | 2017, mis en ligne le 01 juin 2020, consulté le 02 janvier 2021. URL : http://journals.openedition.org/communicationorganisation/5520 ; DOI : https://doi.org/ 10.4000/communicationorganisation.5520 


\title{
Les cadres et le numérique: cultures informationnelles et sentiment d'efficacité
}

\author{
Aurélia Dumas ${ }^{1}$, fabienne Martin-Juchat ${ }^{2}$ et Julien Pierre ${ }^{3}$
}

\section{Introduction}

L'augmentation des flux informationnels au sein des organisations pose depuis une vingtaine d'années la question de la capacité cognitive des cadres (ou salariés à responsabilité) ${ }^{4}$ à les traiter afin de rester efficaces dans leur travail alors même que leurs compétences métiers reposent entre autres sur de telles composantes. Or, Brigitte Guyot soulignait dès 2006 qu'il importe d'interroger de plus près le postulat suivant lequel les TIC augmenteraient la productivité des salariés, tout en s'attachant à comprendre les usages en matière de traitement de l'information, en vue de vérifier la pertinence d'une telle articulation (Guyot 2006, 2013). Nous nous sommes demandé si les cultures de l'information des cadres conditionnent leur sentiment d'efficacité en termes de traitement de l'information (Liquète 2014). Dans la continuité des travaux de Claude Baltz (1998), nous entendons le terme de « cultures de l'information » dans le sens de cultures informationnelles déclinables en compétences, savoir-être et savoir-faire individuels et/ou collectifs dans la manière de gérer les informations (Simonnot 2009).

Au-delà des distinctions existant au cœur du débat entre les conceptions françaises et anglo-saxonnes vis-à-vis du champ de recherche voire de la discipline qui se construit autour de l'information literacy (Boustany, 2014 : 151), notre propos consiste ici à mettre en évidence la manière dont s'imbriquent des aptitudes et des compétences informationnelles au sein des organisations, justifiant dans les termes de Monica Mallowan d'une approche dite transculturelle (Mallowan, 2012, 2014 : 171). En effet, nous nous sommes

1 Aurélia Dumas, Docteure en Sciences de l'information et de la communication, GRESEC, Université de Grenoble - Alpes, 38130 Echirolles ; Aurelia.dumas@iut2.upmf-grenoble.fr

2 Fabienne Martin-Juchat, Professeure des Universités en Sciences de l'information et de la communication, GRESEC, Université de Grenoble - Alpes, 38130 Echirolles ; Fabienne.martin-juchat@univ-grenoble-alpes.fr 3 Julien Pierre, Enseignant-chercheur en Sciences de l'information et de la communication, Audencia Business School, 44000 Nantes ; Julienpierre@audencia.com

4 Nous nous appuyons ici sur la nomenclature établie par l'INSEE. 
intéressés plus spécifiquement à la manière dont des cadres, en fonction de leurs secteurs d'activité d'appartenance, composent avec des applications numériques conçues par des industriels qui s'appuient sur l'affect pour capter l'attention (Martin-Juchat et Staii 2016). Autrement dit, quel(s) rapport(s) de médiation les cadres construisent-ils avec une information en circulation (Baltz 1998), telle que celle issue notamment d'applications numériques grand public (LinkedIn, Facebook, etc.) ?

Dans le cadre d'un projet de recherche sur les usages et les pratiques du numérique, nous avons mené deux premières années d'observation auprès d'étudiants (en majorité issus de formation $\mathrm{Bac}+4$ et +5 ) avec comme question de départ : la relation affective que les étudiants construisent avec les outils numériques (téléphone et ordinateur portable) conditionne-t-elle leur culture informationnelle et détermine-t-elle leurs pratiques et usages en la matière, une fois intégré le monde du travail ? Il est ressorti de ces années de recherche, précédemment publiées (Martin-Juchat, Dumas et Pierre 2015, 2017), la confirmation de tendances partagées par d'autres chercheurs, telles que l'usage intensif des TIC (Badillo et Roux 2015) ou encore des situations de dispersion voire de débordement au travail (Datchary 2011), mais également une ambivalence affective forte entre plaisir et (auto-) contrainte dans le rapport au numérique.

Un résultat a également retenu notre attention : leur sentiment d'efficacité se trouve lié à la capacité à maîtriser les flux informationnels, elle-même corrélative à une faculté de contrôle de la dépendance affective aux outils ainsi qu'au fait de s'imposer des règles explicitables de structuration des flux. Dans la continuité de ces résultats et face aux constats de situations de surcharge voire d'épuisement d'ordre affectif et cognitif des cadres (Felio 2015), notre objectif scientifique a alors consisté à étudier la manière dont ce type d'usagers, une fois devenus salariés, parviennent à rester performants et productifs, pour certains malgré les habitudes antérieures prises, inscrites dans des logiques de débordement, ainsi que nous le verrons, par manque de culture informationnelle acquise à travers la formation scolaire, universitaire ou encore professionnelle, dont une composante nous apparait comme essentielle à savoir la gestion des ressources affectivo-cognitives au cœur du traitement de l'information.

Nous formulons l'hypothèse que les cadres qui manifestent un sentiment d'efficacité ont développé une culture informationnelle tacite et plus ou moins partagée en fonction de la (des) culture(s) organisationnelle(s). Dans une logique de co-construction de notre démarche de recherche avec les acteurs professionnels, notre questionnement ainsi que notre méthodologie de recherche ont été élaborées en collaboration avec un partenaire professionnel (la start-up Beebuzziness). À la suite de cette phase d'enquête préliminaire, plus d'une trentaine d'entretiens de recherche (d'une durée moyenne d'une heure), suivant une approche qualitative, a été réalisée auprès de cadres, en 
vue de valider nos propositions de recherche formulées. Notre échantillon a été construit en fonction de 5 panels constitués selon les types d'organisation d'appartenance des cadres interrogés à savoir le secteur du conseil, la start-up, la PME, la grande entreprise et la fonction publique. Le guide d'entretien mis au point a donc visé à questionner, en fonction des différents types d'organisation étudiés, le poids ainsi que les spécificités des cultures informationnelles identifiées dans la gestion de l'information individuelle et collective des cadres, dont nous allons voir qu'ils se trouvent au cœur de la construction du sentiment d'efficacité au et dans le travail.

\section{Les consultants ou comment s'extraire du flux pour pouvoir être efficace}

Plusieurs caractéristiques communes se retrouvent au sein du métier de consultant, telles qu'une forte mobilité, un lien très fort au client, un besoin en informations de qualité pour rédiger des propositions de prestations et un travail minoritairement en équipe. Alors que dans aucune des organisations des consultants de notre panel, des règles n'ont été mises en place pour la gestion des flux, il est marquant d'observer le filtrage massif que ces derniers opèrent. Une des formes de contrôle des flux mis en ouvre est la quasiabsence de présence sur les réseaux sociaux, hormis LinkedIn pour les seniors. Massivement, les consultants déclarent que ces services n'amènent aucune valeur ajoutée et se révèlent chronophages.

Durant les premières années de consulting, la tendance à vouloir essayer de gérer simultanément des flux privés et professionnels, via l'intrusion des outils de captation de l'attention (Martin-Juchat et Staii 2016), tend à voler en éclats dès que la vie privée se complexifie. La conjonction de ces flux et de l'impossibilité de les gérer a amené certains consultants jusqu'à quitter le métier. Dès que la vie personnelle prend davantage de place, la fatigue, subséquente au nombre pléthorique de sollicitations, peut devenir ingérable et d'aucuns se voient alors dans l'incapacité de poursuivre un tel rythme.

Aussi la capacité à gérer les flux est-elle essentielle dans le sentiment d'efficacité au travail. Néanmoins, il apparaît que les flux issus de la sphère professionnelle restent, dans ce secteur, très "gérables", à condition et du fait de filtres apposés, avec environ 50 mails reçus par jour, dont 5 importants à traiter. Beaucoup de SMS sont envoyés et reçus mais ce moyen de communication est perçu comme plus efficace, car moins intrusif, pour une grande majorité des consultants interrogés. Tous ont mis en place des règles de structuration de leurs flux précises et pensées: ils peuvent tous justifier de la manière dont ils utilisent le mail, le téléphone et le SMS. Pour l'ensemble des consultants, il s'agit de trouver des espaces de concentration au cours de leur journée et toutes les techniques sont bonnes pour s'isoler: ils verrouillent et filtrent les flux, ne gèrent que l'urgent que ce soit du côté des clients comme de la vie 
privée. Ils parviennent alors, dans ces temps de concentration qu'ils ménagent, à filtrer les sollicitations superfétatoires, en dehors des urgences.

Tous ceux qui perdurent dans le métier présentent donc un autocontrôle très fort, des tactiques pour minimiser les flux, des techniques très précises pour les structurer, en d'autres termes, développent une culture informationnelle, qui permet l'appropriation des flux d'information entrants et sortants.

Les collègues sont rendus complices, tout comme la famille. Il apparait à cet égard que les consultants, qui nourrissent un fort sentiment d'efficacité dans leur gestion des flux, présentent également un capital de confiance en eux qui leur permet de faire comprendre qu'il ne faut pas les déranger, qu'ils doivent s'isoler. Pour se dégager du temps à haute valeur ajoutée intellectuelle, ces derniers n'hésitent pas à débloquer des demi-journées ou des temps en dehors des heures de travail. La solidarité familiale est alors inévitable. Tenir dans ce métier n'est possible que s'il existe une complicité de la part des proches. Car pour trouver dans leur journée ces temps à haute valeur ajoutée intellectuelle, les consultants interrogés déclarent tous travailler en dehors du bureau et, surtout, en dehors des heures de travail. Les heures en présentiel sont consacrées à de la gestion de la relation. Certains parlent de "zapping relationnel», durant la journée, en particulier par le biais des outils numériques et du téléphone.

À ce titre, la pression affective se révèle forte chez les jeunes consultants qui, par désir de s'insérer et d'être reconnus professionnellement, présentent alors des difficultés et des réticences à réguler les flux d'information. Une fois leur légitimité acquise et leur place faite, ces derniers se sentent moins inquiétés par des questions de reconnaissance au travail et peuvent oser refuser de répondre à la sollicitation. En d'autres termes, le sentiment d'efficacité entre largement en corrélation avec la capacité à gérer les flux, qui elle-même est liée aux ressources affectives des individus. Tous les consultants interrogés reconnaissent l'importance d'Internet en tant qu'outil de veille ainsi que celle des outils numériques d'un point de vue de la gestion des informations, $a$ contrario des réseaux sociaux qui sont rarement utilisés car considérés comme à faible valeur ajoutée. Tous se disent également dépendants du numérique et l'assument sans problème : dans ce contexte de "bain numérique ", le plaisir reste majoritaire, au-delà de la contrainte librement assumée.

\section{Les start-ups ou comment articuler vie privée et vie professionnelle grâce au numérique}

Deux types de contextes culturels tendent à ressortir des résultats obtenus : certains entrepreneurs manifestent une intégration entre leur activité professionnelle et un cercle de proches tandis que d'autres se retrouvent bien plus isolés socialement. Si les configurations de travail sont très différentes dans l'organisation de l'espace (open space, baies vitrées, entrepôt réaménagé), le système d'information, la stratégie de développement de l'entreprise 
ou encore dans la gestion des emplois du temps ou l'allocation de fonds personnels dans l'entreprise, la principale récurrence observée vient d'un goût de l'entreprenariat, corrélatif à un engagement participant d'un effacement de la barrière vie professionnelle/vie privée.

On peut noter que ceux qui ont des espaces ouverts sont ceux qui déclarent le plus de flux (+500 par jour), mais le faible score d'efficacité tient moins à des perturbations attentionnelles qu'à l'intensité de leur engagement dans les flux : la surexcitation et la fatigue ne seraient pas l'effet d'un environnement bruyant, mais le choix d'une manière de travailler, d'organiser son quotidien, de prioriser les rapports entre le domaine privé et professionnel, autrement dit de l'ordre d'un style de vie. En effet, les entrepreneurs travaillent majoritairement en famille ou entre amis, et le numérique permet cette proximité.

La culture de l'outil est donc très développée. Aucun des entrepreneurs n'utilise le même outil (à l'exception notable de Slack ${ }^{5}$ ), mais celui qui est retenu se révèle complètement optimisé et automatisé. Les équipes s'entendent autour des modalités d'interaction et de gestion par le numérique. Elles vont ensuite prescrire ces modalités à leurs partenaires. Le «bien-être au travail» semble alors appartenir à celui qui dicte les règles de l'attention. En effet, l'usage des outils est relié à une discipline forte de cloisonnement des canaux privés/personnels et professionnels, à une hygiène informationnelle, également adressée aux proches, aux collaborateurs, aux prestataires et aux clients. C'est un paradoxe intéressant par rapport au point précédent : le plaisir au travail vient d'une proximité affective avec le lieu ou les collaborateurs, mais les outils numériques permettent de tenir à distance cette proximité.

Aussi les personnes interrogées au sein de cette communauté se rejoignentelles dans un travail de réflexivité autour des pratiques informationnelles car intrinsèquement liées à leur écologie familiale et professionnelle.

\section{Les cadres en entreprise ou comment l'initiative managériale se trouve au cœur de la gestion des flux}

Les salariés interrogés, issus de grandes entreprises ou de PME, ont présenté des conditions de travail diverses et la variété des cultures organisationnelles observées ne s'est pas révélée indexée à la taille de l'entreprise d'appartenance mais bien plutôt à l'initiative managériale en matière de règles de fonctionnement et outils mis en place dans la gestion des flux infocommunicationnels. Si la majorité des salariés interrogés travaillent dans des open space, alors que certains seulement possèdent un bureau isolé, de nombreux déplacements professionnels jalonnent leur emploi du temps et limitent leur

5 Slack est un service web et une application, dont le slogan est «be less busy " : l'outil s'interface avec une multitude d'autres services en ligne (gestion clients, mails, serveurs, comptes bancaires, logistique, etc.). Il est possible alors de définir des scripts de notification selon la provenance, la fréquence, la présence de mots-clés et d'adresser un suivi à l'intention des membres de l'équipe. Dans certaines entreprises, la maîtrise de Slack est une compétence attendue dans les offres d'emploi et évaluée dans la phase de recrutement. 
temps de présence au bureau. Dès lors, plusieurs bénéficient de possibilités de télétravail sur certains jours dédiés dans la semaine, généralement formalisées par un accord d'entreprise ou encore des conventions collectives. Pour tous, la relation avec les clients apparaît comme primordiale dans leur quotidien de travail et conditionne leur gestion des flux, obligeant à donner priorité au(x) client(s) sur toute autre activité professionnelle.

Pour la majorité des salariés interrogés, il n'existe pas de règles organisationnelles fixées de manière formelle par l'entreprise en matière d'utilisation et de gestion des outils numériques. Pour autant, apparaissent des modes de fonctionnement implicites dictées par l'entreprise, qui se révèlent déterminants dans la culture informationnelle des salariés. De tels cadres opèrent à échelle individuelle et collective, participant d'une culture organisationnelle partagée, mais rencontrent également les aptitudes et tactiques développées par chacun dans leur gestion courante des flux d'information. Nous sommes donc dans ces organisations au stade du « faire avec » (Cordier et Liquète $2014: 83$ ).

Il est question pour les salariés interrogés de réguler les flux entrants et sortants, au travers, notamment, d'un filtrage et d'une hiérarchisation des contenus suivant leur importance et leur valeur ajoutée dans le travail. Il s'agit de parvenir à ne pas se laisser déborder par la gestion des flux pour maintenir un niveau d'efficacité dans le travail quotidien et intégrer l'utilisation des TIC à cette productivité. Ces derniers reçoivent, pour la plupart, en moyenne, entre 20 à 50 mails ainsi que cinq à dix coups de téléphone par jour. Or, sur l'ensemble des flux traités, tout type de support confondu, seuls quelques-uns sont considérés comme importants et nécessitent de leur part une attention particulière et une réponse immédiate. Certains favorisent l'utilisation des SMS, d'autres des mails ou du téléphone, d'autres encore des messageries instantanées. Ces choix tendent à être dictés par les modes de fonctionnement dominants dans l'entreprise, mais également par des tactiques individuelles de distribution des outils en fonction des interlocuteurs (clients, responsables hiérarchiques, collègues, famille, etc.). La distribution des fonctions conférées aux différents types de support utilisés permet alors de cloisonner les activités et entend maintenir la concentration nécessaire dans le travail. Dans le même ordre d'idée, un aménagement de l'emploi du temps, qui alterne temps de présence au bureau et télétravail, est mis en place par certains salariés dans une visée stratégique de régulation des sollicitations, permettant un traitement plus en profondeur des informations : les temps de travail à domicile permettent ainsi aux salariés de s'isoler et de s'extraire du bruit et de l'agitation ambiante, notamment dans le cadre de l'open space, en vue de gagner en efficacité dans le travail. À l'instar des deux précédents panels, la majeure partie des salariés interrogés ont adressé des règles à leur entourage professionnel mais aussi personnel. Il peut également s'agir de règles tacites instaurées entre les personnes par la force de l'habitude. 
En outre, à défaut d'une régulation des usages et des pratiques à une échelle organisationnelle, certains ont également décidé de promouvoir très explicitement des modes de fonctionnement auprès de leur équipe. Un des cadres interrogés a établi auprès des salariés qu'il manage une procédure précise en matière de gestion des mails et a instauré une charte d'écriture qui fonctionne comme un langage partagé, en vue de favoriser un accès rapide aux informations. Autrement dit, si l'organisation elle-même ne tend pas à édicter des règles collectives en matière de gestion des flux info-communicationnels, des modes de régulation d'ordre collectif apparaissent au sein de certaines équipes sous l'impulsion managériale. Néanmoins, il apparaît que ces règles ne tendent pas à être appliquées dans le cadre des interactions avec les clients dans la mesure où est attendue une forte disponibilité et réactivité vis-à-vis de ces derniers.

Enfin, il ressort presque unanimement un sentiment d'accroissement de l'efficacité au travail dans l'utilisation des TNIC, allant de pair avec la perception que cette dernière n'a pas fait baisser leur productivité. La performance se ressent plus particulièrement du côté des facilités offertes en matière de recherche et de traitement de l'information, du fait notamment de la dématérialisation des supports. Si certains s'avouent volontiers dépendants des TNIC, rendant par là même poreuse la frontière entre les temps de travail et les temps personnels, ils témoignent massivement d'un sentiment de plaisir dans leurs usages.

Néanmoins, la majorité des cadres interrogés se disent confrontés à une surcharge informationnelle et certains se plaignent d'une instantanéité inutile et chronophage des échanges, qui nuit selon eux à l'appropriation des informations réellement importantes pour l'activité de travail. A cet égard, l'exigence de la relation client au regard de la disponibilité attendue de la part du salarié, qu'un des cadres interrogés qualifie de "perversion d'utilisation " des outils du numérique, tend à participer de l'instauration d'un sentiment d'inefficacité dans le travail. S'observe ici la manière dont une culture organisationnelle qui donne la priorité à la relation client demande aux salariés de développer des aptitudes affectives dans la capacité à gérer les flux.

\section{Le secteur de la fonction publique ou comment tracer son chemin dans un environnement hiérarchisé}

Tous les agents contactés partagent un environnement très hiérarchisé, composé de multiples entités, éclatées sur de nombreux sites, avec des protocoles d'administration et de communication très lourds et la mise à disposition de nombreux logiciels peu ergonomiques. Beaucoup sont nouveaux dans leur poste et ont dû s'adapter rapidement à la culture informationnelle de leur institution. Tous ont été confrontés à une réorganisation des services internes. Certains des responsables disposent de marges de manœuvre pour transformer leur organisation par le numérique, mais le cas échéant, il ne 
s'agit jamais d'une décision stratégique provenant de la hiérarchie, mais bien d'un choix personnel. Pour les autres cadres interrogés (en fonction dans des services de relations internationales ou dans la gestion des fonds documentaires par exemple), il n'y a soit pas la conscience de la nécessité de ce type de stratégie, soit pas le courage de la mettre en œuvre, notamment en raison de représentations négatives fortes sur l'organisation, son inertie et l'inadéquation d'une politique numérique en regard des usages. Enfin, certains cadres de la fonction publique se trouvent dans des processus informationnels extrêmement standardisés par les services de l'État et n'ont pas d'initiative sur la transformation numérique de leur service.

Les flux d'information se révèlent similaires dans les différents services de la fonction publique étudiés (une moyenne de 100 mails par jour en réception, dont environ $10 \%$ sont dits importants). Certains process métiers sont perçus comme très chronophages, tels que la veille informationnelle permanente dans les bibliothèques, et empiètent inconsidérément sur l'organisation du quotidien de travail. Il est à noter que certains cadres interrogés, anciennement salariés dans le secteur privé, déclarent bien plus nombreux les flux d'information dans le public. Certains peinent à prioriser les flux et se trouvent submergés.

Pour éviter la dispersion et se coordonner, la présence de règles, de consignes partagées se fait systématique mais correspond à un niveau individuel, de l'ordre de "bricolages ». Nombreux sont à la recherche de conseils pour optimiser leur temps avec le numérique. Mais, rien n'est mis en place au niveau institutionnel : la DSI n'intervient pas dans les usages.

$\mathrm{Au}$ sein de ce panel, la maîtrise de la dispersion entre le privé et le professionnel est au mieux assumée : elle relève d'une culture acceptée par les interactants, à l'intérieur de laquelle une démarche réflexive permet de construire des compétences. Au pis, cette maîtrise est illusoire : on note, en effet, un décalage fort au sein d'un même discours entre une prise de distance et des pratiques intensives. Une phase d'observation nous aurait permis d'établir plus finement le hiatus entre le déclaratif et le réalisé.

Quelles que soient les règles mises en place, le niveau de conscience de cette dispersion peut être corrélé avec le sentiment d'efficacité. En effet, ceux qui estiment être efficaces grâce au numérique sont ceux qui - paradoxalement - ont des modalités de gestion problématiques : le numérique étant omniprésent dans leurs activités, ils auraient tendance à le survaloriser sans se rendre compte des difficultés que cela génère - en fait, ils sont débordés, toujours en retard ou toujours engagés dans le numérique même dans les temps de repos ou de famille.

\section{Conclusion}

Tel que nous l'avons łobservé par le biais de nos entretiens, rares sont les « tribus » professionnelles (Baltz 1998) qui partagent collectivement un savoirfaire des outils intégrant des logiciels de gestion de l'information. Or, dans les 
cas d'absence de tactiques de gestion des flux informationnels, apparaît un risque d'épuisement des ressources cognitivo-affectives et d'improductivité dans l'activité de travail.

Le sentiment d'efficacité au travail est corrélatif à la maîtrise affective des outils. Nous avons ainsi observé l'émergence d'un " travail émotionnel » (Hochschild 1983, 2003) se faisant aptitudes émotionnelles, dans la manière de gérer les ressources numériques, en fonction des contextes et des cultures organisationnels associés : aptitudes et non compétences, car à ce jour absentes des référentiels dans les organisations, elles participent d'un travail invisible réclamé aux cadres par l'organisation sans pour autant être explicitées donc reconnues. Par contre, il s'agit bien, à la lecture des résultats, de souligner que ces aptitudes, dont fait partie la maîtrise des émotions, conditionnent la capacité à gérer les flux informationnels. À ce titre, les réseaux sociaux grand public sont très couramment perçus par les salariés interrogés comme sources de dispersion et de perte de temps, leur demandant de développer de fortes aptitudes à l'autocontrôle.

Le sentiment d'efficacité au travail se trouve donc en partie lié aux capacités à gérer les flux et, plus spécifiquement, à les réguler dans un contexte de porosité voire de disparition des frontières entre vie professionnelle et vie privée (Broadbent 2011). Or, peu d'organisations ont construit une culture de l'information, formalisée par des règles de fonctionnement collectives en matière de gestion des flux, des usages et pratiques de connexion ou de déconnexion, en vue notamment de protéger la vie privée du salarié face aux risques d'hyperconnexion. À défaut d'un encadrement des pratiques pensé et pris en charge par l'organisation elle-même, les cadres interrogés présentent des tactiques, assumées et précises, de l'ordre du "bricolage » individuel, du faire avec (Cordier et Liquète 2014 : 83), pour faire face au flux info-communicationnel. Est-il cependant possible de penser la montée en compétences de ces bricolages?

Avant même la question de la finalité de la nécessité de former et ce, dès l'entrée dans l'enseignement supérieur, à une culture de l'information, une approche en termes d'information transculturelle ou de translittératie (Delamotte, Liquète et Frau-Meigs 2014 ; Mallowan 2014), qui tenterait de construire des ponts entre les usages des outils, la gestion des sources d'information, les habiletés à s'en saisir, les compétences en termes de savoirêtre, dont les aptitudes émotionnelles font partie, se révèle plus que nécessaire. Chemin faisant, il nous faudrait alors envisager que les évolutions conduisant à un passage du côté des compétences informationnelles au sein des organisations induisent également des systèmes d'évaluation, susceptibles de produire de nouvelles formes de domination mais également de distanciation et d'émancipation. 


\section{BIBLIOGRAPHIE}

BADILLO Patrick-Yves et ROUX Dominique (dir.), 2015, Le numérique pour enseigner autrement, Paris, Economica.

BALTZ Claude (1998), "Une culture pour la société de l'information ? Position théorique, définition, enjeux ", Documentaliste-sciences de l'information, volume $35, \mathrm{n}^{\circ} 2$, p. $75-82$.

BOUSTANY Joumana, 2014, «Le champ de l'information literacy en France à travers les publications scientifiques ", Hermès, p. 139-158.

BROADBENT Stefana, 2011, L'intimité au travail. La vie privée et les communications personnelles dans l'entreprise, Paris, FYP Editions.

CORDIER Anne et LIQUÈTE Vincent, 2014, «La culture générale face à l'information ", Hermès, p. 69-89.

DATCHARY Caroline, 2011, La dispersion au travail, Toulouse, Octarès.

DELAMOTTE Eric, LIQUÈTE Vincent et FRAU-MEIGS Divina, 2014, « La translittératie ou la convergence des cultures de l'information : supports, contextes et modalités ", Spirale, p. 145-156.

FELIO Cindy, 2015, «Les stratégies de déconnexion des cadres équipés en TIC mobiles ", Nouvelle revue de psychosociologie 2015/1, n 19, p. 241-254.

GUYOT Brigitte, 2006, Dynamiques informationnelles dans les organisations, Paris, Hermès.

GUYOT Brigitte, 2013, " Place et enjeux de l'information dans les organisations », dans V. Clavier et C. Paganelli (dir.), L’information professionnelle, Paris, Lavoisier, p. 199217.

HOCHSCHILD Arlie R., 1983, The managed heart: the commercialization of human feeling, Berkeley, The University of California Press.

HOCHSCHILD Arlie R., 2003, "Travail émotionnel, règles de sentiments et structure sociale », Travailler, 1, 9, p. 19-49.

LIQUÈTE Vincent (dir.), 2014, « Cultures de l'information », Hermès, Paris, CNRS Éditions.

MALLOWAN Monica, 2012, "Intelligence et transculture de l'information ", Communication EOrganisation, $\mathrm{n}^{\circ} 42,2012$, p. 27-48.

MALLOWAN Monica, 2014, « La transculture de l'information : vers une posture multiculturelle de l'information », Hermès, p. 159-175.

MARTIN-JUCHAT Fabienne, DUMAS Aurélia et PIERRE Julien (2015), «Vers des bricolages stratégiques pour faire face à l'ambivalence affective du rapport au numérique », dans B. Meyronin (dir.), La génération Y, le manager et l'entreprise, Grenoble, PUG, p. 29-72.

MARTIN-JUCHAT Fabienne, DUMAS Aurélia et PIERRE Julien, 2017, "Logiques affectives des usages non pédagogiques du numérique en situation de cours ", Numérique et Education, Questions de communication, série actes, $\mathrm{n}^{\circ}$ 34, p. 211-226. 
MARTIN-JUCHAT Fabienne et STAII Adrian (dir.) (2016), Industrialisation des émotions et radicalisation de la modernité, Paris, L'Harmattan, collection « Communication et civilisation".

SIMONNOT Brigitte, 2009, «Culture informationnelle, culture numérique : au-delà de l'utilitaire », Les Cahiers du numérique, 3/2009, Volume 5, p. 25-37.

Résumé : Notre recherche pose la question de la corrélation entre sentiment d'efficacité au travail et cultures de l'information. Par des entretiens approfondis, nous avons cherché à saisir les bricolages et aptitudes développés au travail, selon différentes cultures organisationnelles (consultants, start-ups, PME et grandes entreprises, fonction publique). Nous nous sommes intéressés spécifiquement à une catégorie de cadres supérieurs confrontés à des situations régulières de surcharge informationnelle. Les pratiques qui se dégagent tendraient à constituer, voire à consolider des cultures informationnelles propres au sein desquelles l'aptitude à maîtriser affectivement le rapport au numérique est une composante essentielle de la recherche d'efficacité professionnelle. Cependant, le passage d'aptitudes à compétences reste à interroger, aussi bien pour les organisations qu'en termes d'enjeux sociétaux.

Mots-clés : cultures informationnelles, cadres, gestion des flux informationnels, sentiment d'efficacité au travail, cultures organisationnelles.

\begin{abstract}
Our research raises the question of the correlation between feeling of efficiency at work and information cultures. Through in-depth interviews, we sought to capture the crafts and skills developed at work, according to different organizational cultures (consultants, startups, SMEs and large enterprises, civil service). We are specifically interested in a category of senior managers to regular situations of information overload. The practices that emerge tend to constitute or even consolidate specific information cultures in which the ability to control affectively the relationship to the digital is an essential component of the search for professional efficiency. However, the transition from skills to competences remains to be questioned, both for organizations and in terms of societal issues.
\end{abstract}

Keywords: informational cultures, executives, management of information flows, feeling of efficiency at work, organizational cultures. 